\title{
A Graphical User Interface for Scattering Analysis of Electromagnetic Waves Incident on Planar Layered Media
}

\author{
Ali Mirala ${ }^{1^{*}}$ and Ali Abdolali ${ }^{2}$ \\ ${ }^{1}$ Department of Electrical Engineering, Amirkabir University of Technology (Tehran Polytechnic), Tehran, Iran \\ ${ }^{2}$ Department of Electrical Engineering, Iran University of Science and Technology, Tehran, Iran \\ *corresponding author, E-mail: mirala@aut.ac.ir
}

\begin{abstract}
This paper introduces a MATLAB-based Graphical User Interface (GUI) which could help electromagnetics engineers and researchers who are interested in designing layered media for various applications. The paper begins with presenting the analysis method the program employs, continues by encountering specific considerations and techniques of implementation, and ends with providing different numerical examples. These examples show good efficiency of the program for analysis of diverse problems.
\end{abstract}

\section{Introduction}

Layered or composite Media have drawn lots of attention due to their wide spectrum of applications. They could serve as microwave and optical filters [1,2], coatings for cloaking and radar cross section (RCS) reduction [3-6], boosted transparency surfaces [7], high-reflection coatings [8], multilayer circuits [9], etc. This fact has not only resulted in myriads of researches pertaining their usages, but also has inspired many novel analysis methods, some of which are: transfer matrix method (TMM) [10,11], classical numerical techniques such as finite difference time domain (FDTD) [12] and integral equations [13], transmission line equivalence [14], and recursive methods [15,16].

This paper uses a relatively simple and efficient method which is based on transfer matrix method. The mathematical formulation is elaborated in [6] and will be restated briefly here with stressing some practical considerations. The reason we choose the transfer matrix method is firstly for its generality to solve nearly any related homogeneous theoretical and practical structure and secondly, for its clear and well-developed algorithm to be converted into MATLAB code. The provided algorithm is applicable for lossless and lossy media, anisotropic as well as isotropic ones, dispersive media, and complex structures composed of metamaterials and conventional media. The only restriction is that each layer should be homogeneous in every direction. Of course in special cases which the inhomogeneity is aligned with the structure's normal vector (here $\mathrm{z}$ axis), the structure may be viewed as infinite number (in practice, large number) of homogeneous layers and analyzed using current program. The proposed algorithm employs matrices and so is suitable to be realized by MATLAB which has a matrixbased coding.

The program receives constitutive parameters of structure layers and incident wave properties namely frequency, incidence direction and polarization and gives the scattered wave parameters. The incident and scattered waves are all assumed to be planar in this research. After describing mathematical formulation, it will be verified using a few significant numerical examples.

\section{Formulation}

The geometry of the structure and incident and scattered wave vectors are shown in figure 1 . The wave travels from left halfspace to the right halfspace that are assumed to be air (or some other isotropic media). Also, the right halfspace may be perfect electric conductor (PEC). This case is especially important when the structure is supposed to be used as a radar absorbing material (RAM) or anti-reflection coating.

Each homogeneous layer in its most general form can be identified with its permittivity and permeability tensors (which are frequency dependent in common) and its thickness. We do not impose any limitation for permittivity and permeability tensors' elements in order to keep the generality of the problem. However, mostly in the literature, these tensors are assumed diagonal. The physical limitation that this assumption impose is that the layer's principal axis should be aligned with the normal vector of the structure.

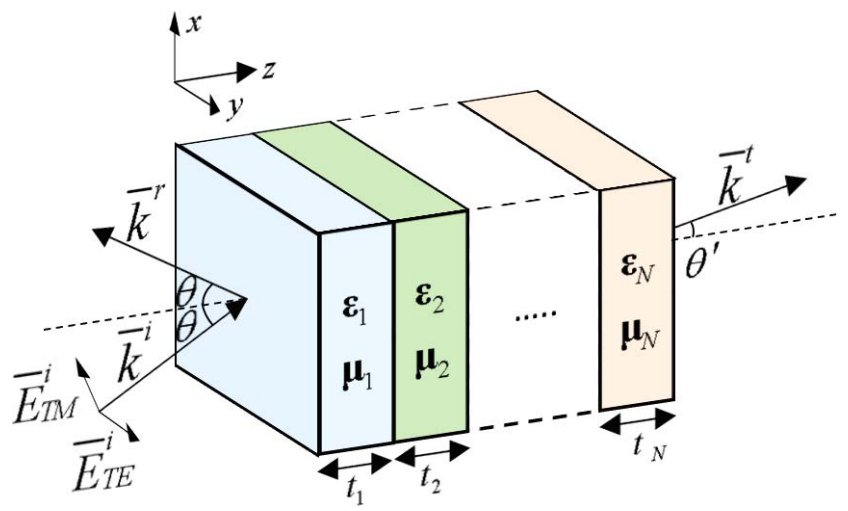

Figure 1: Geometry of the problem 
Note that the propagation direction lies in the $\mathrm{x}-\mathrm{z}$ plane in figure 1 and this will be applied throughout our formulation. However, this does not reduce the generality of the problem as far as our formulation contains the general form of permittivity and permeability tensors. That's because if the propagation be not in $\mathrm{x}-\mathrm{z}$ plane, we can take it in $\mathrm{x}-\mathrm{z}$ plane and instead, rotate the whole structure around the $\mathrm{z}$ axis in order to keep the relative angle untouched. To apply such rotation, tensors should be updated by $\boldsymbol{\varepsilon} \rightarrow \mathbf{R}_{z}^{-1}(\varphi) \boldsymbol{\varepsilon} \mathbf{R}_{z}(\varphi) \quad$ and $\quad \boldsymbol{\mu} \rightarrow \mathbf{R}_{z}^{-1}(\varphi) \boldsymbol{\mu} \mathbf{R}_{z}(\varphi)$

conversions in which $\boldsymbol{\varepsilon}, \boldsymbol{\mu}, \mathbf{R}_{z}$, and $\varphi$ are the relative permittivity, relative permeability, rotation matrix about $\mathrm{z}$ axis, and propagation angle, respectively.

To know the overall scattering behavior of the structure, we should learn how planar waves propagate in any medium possessing aforementioned parameters. Assuming all plane wave fields as harmonic functions of space and time as $\exp (j \omega t-j \bar{k} \cdot \bar{r})$, Maxwell's curl equations in frequency domain become $\bar{k} \times \bar{E}=\omega \bar{B}$ and $\bar{k} \times \bar{H}=-\omega \bar{D}$ which in combination give the wave equation as:

$$
\bar{k} \times\left(\boldsymbol{\mu}^{-1}(\bar{k} \times \bar{E})\right)+k_{0}^{2} \boldsymbol{\varepsilon} \bar{E}=0
$$

where $k_{0}$ is the wave number in free space. Equation (1) is a vector equation and could also be represented by matrix form as:

$$
\left(\mathbf{\kappa} \boldsymbol{\mu}^{-1} \mathbf{\kappa}+k_{0}^{2} \boldsymbol{\varepsilon}\right) \bar{E}=0
$$

where $\boldsymbol{\kappa}$ is a matrix that transforms any vector like $\bar{E}$ to $\bar{k} \times \bar{E}$ and its value is:

$$
\mathbf{\kappa}=\left(\begin{array}{ccc}
0 & -k_{z} & 0 \\
k_{z} & 0 & -k_{x} \\
0 & k_{x} & 0
\end{array}\right)
$$

while the value of $k_{x}$ is the same in the whole space due to phase matching at the boundaries and equals to $k_{x}=k^{i} \sin (\theta)$ in which $k^{i}$ is the wave number in the left halfspace and $\theta$ denotes the incident wave propagation angle .

Equation (2) is the matrix wave equation and in order to yield nonzero solution for electric field, $\boldsymbol{\kappa} \boldsymbol{\mu}^{-1} \boldsymbol{\kappa}+k_{0}^{2} \boldsymbol{\varepsilon}$ should be singular. This condition leads us to equation:

$$
\left|\mathbf{\kappa} \boldsymbol{\mu}^{-1} \mathbf{\kappa}+k_{0}^{2} \boldsymbol{\varepsilon}\right|=0
$$

which is a fourth order equation in terms of $k_{z}$ and has four solutions in general. Therefore, there are four wave vectors each of which expresses a different propagation mode. The overall field inside the medium is the linear superposition of all modes' fields as:

$$
\bar{E}=\sum_{m=1}^{4} E_{m} \hat{E}_{m} e^{-j \bar{k}_{m} \cdot \bar{r}}
$$

where $E_{m}$ and $\hat{E}_{m}$ are the electric field excitation coefficient and unit vector of the mode " $m$ " respectively and $\bar{k}_{m}=k_{x} \hat{x}+k_{z m} \hat{z}$ is the wave vector of that mode. The excitation coefficients are determined using the boundary conditions. To calculate the unit vectors, we return to equation (2). This matrix equation is in fact a set of three linear equations for $\bar{E}$ elements. Each of these equations is equation of a plane in $\left(E_{x}, E_{y}, E_{z}\right)$ space and the three planes intersect in one line that passes from origin and on which the $\bar{E}$ solution lies. Thus, the electric unit vector is the same as the unit direction vector of the line and equals to the normalized cross product of planes' normal vectors. In sum, assuming $\left(\boldsymbol{\kappa} \boldsymbol{\mu}^{-1} \mathbf{\kappa}+k_{0}^{2} \boldsymbol{\varepsilon}\right)_{r}$ to be the r'th row of the matrix, the unit electric field would be:

$$
\hat{E}=\frac{\left(\kappa \mu^{-1} \boldsymbol{\kappa}+k_{0}^{2} \boldsymbol{\varepsilon}\right)_{r} \times\left(\boldsymbol{\kappa} \boldsymbol{\mu}^{-1} \boldsymbol{\kappa}+k_{0}^{2} \boldsymbol{\varepsilon}\right)_{r^{\prime}}}{\left|\left(\boldsymbol{\kappa} \boldsymbol{\mu}^{-1} \boldsymbol{\kappa}+k_{0}^{2} \boldsymbol{\varepsilon}\right)_{r} \times\left(\boldsymbol{\kappa} \boldsymbol{\mu}^{-1} \boldsymbol{\kappa}+k_{0}^{2} \boldsymbol{\varepsilon}\right)_{r^{\prime}}\right|}
$$

for any $r$ and $r^{\prime}$ if $r \neq r^{\prime}$. The value of above equation does not depend on the choice of $r$ and $r^{\prime}$, since three planes intersect in a single line. Equation (6) should be worked out for each $k_{z m}$. The rest of calculations is the ordinary transfer matrix method.

To apply transfer matrix method, we need to define characteristic matrix of each layer that contains all the information about that layer and relates the field values of layer's two sides. The n'th layer characteristic matrix is defined as $\mathbf{C}_{n}=\mathbf{B}_{n} \mathbf{P}_{n} \mathbf{B}_{n}^{-1}$ in which [6,11]:

$$
\begin{gathered}
\mathbf{B}_{n}=\left(\begin{array}{ccc}
\hat{E}_{1 n} \bullet \hat{x} & \hat{E}_{4 n} \bullet \hat{x} \\
\hat{E}_{1 n} \bullet \hat{y} & \cdots & \hat{E}_{4 n} \bullet \hat{y} \\
\boldsymbol{\mu}_{n}^{-1}\left(\bar{k}_{1 n} \times \hat{E}_{1 n}\right) \cdot \hat{x} & \boldsymbol{\mu}_{n}^{-1}\left(\bar{k}_{4 n} \times \hat{E}_{4 n}\right) \cdot \hat{x} \\
\boldsymbol{\mu}_{n}^{-1}\left(\bar{k}_{1 n} \times \hat{E}_{1 n}\right) \cdot \hat{y} & \boldsymbol{\mu}_{n}^{-1}\left(\bar{k}_{4 n} \times \hat{E}_{4 n}\right) \cdot \hat{y}
\end{array}\right) \\
\mathbf{P}_{n}=\exp \left(\begin{array}{ccc}
-j k_{z 1 n} t_{n} & & \\
& \ddots & -j k_{z 4 n} t_{n}
\end{array}\right)
\end{gathered}
$$

The matrix given in equation (7) should be calculated for the left and right halfspaces as well as layers and $\mathbf{B}_{0}$ and $\mathbf{B}_{N+1}$ will stand for them. To do this calculation, we use $\bar{k}_{1}=\bar{k}_{2}=\bar{k}^{i} \quad, \quad \bar{k}_{3}=\bar{k}_{4}=\bar{k}^{r} \quad, \quad \hat{E}_{1}=\hat{E}_{3}=\hat{y} \quad$, 
$\hat{E}_{2}=\cos (\theta) \hat{x}-\sin (\theta) \hat{z}$, and $\hat{E}_{4}=\cos (\theta) \hat{x}+\sin (\theta) \hat{z}$ for the left halfspace and similar relations for the right halfspace.

Next, we define the scattering matrix which relates the incident and scattered waves' fields in such a way that:

$$
\left(\begin{array}{c}
E_{T E}^{t} \\
E_{T M}^{t} \\
0 \\
0
\end{array}\right)=\mathbf{S}\left(\begin{array}{c}
E_{T E}^{i} \\
E_{T M}^{i} \\
E_{T E}^{r} \\
E_{T M}^{r}
\end{array}\right)
$$

and could be calculated as:

$$
\mathbf{S}=B_{N+1}^{-1}\left(\prod_{n=1}^{N} C_{N-n+1}\right) B_{0}
$$

To take advantage of these relations, we define reflection and transmission matrices as:

$$
\Gamma=-\left(\begin{array}{ll}
S_{33} & S_{34} \\
S_{43} & S_{44}
\end{array}\right)^{-1}\left(\begin{array}{ll}
S_{31} & S_{32} \\
S_{41} & S_{42}
\end{array}\right)
$$

and:

$$
\mathbf{T}=\left(\begin{array}{ll}
S_{11} & S_{12} \\
S_{21} & S_{22}
\end{array}\right)+\left(\begin{array}{ll}
S_{13} & S_{14} \\
S_{23} & S_{24}
\end{array}\right) \boldsymbol{\Gamma}
$$

where $S_{i j}$ is the ij'th component of scattering matrix. If the structure is PEC-backed, there is no transmission ( $\mathbf{T}=0$ ) and from equation (11) the reflection matrix turns out to be:

$$
\boldsymbol{\Gamma}=-\left(\begin{array}{ll}
S_{13} & S_{14} \\
S_{23} & S_{24}
\end{array}\right)^{-1}\left(\begin{array}{ll}
S_{11} & S_{12} \\
S_{21} & S_{22}
\end{array}\right)
$$

Now, the scattered fields could be obtained through:

$$
\begin{aligned}
& \left(\begin{array}{l}
E_{T E}^{r} \\
E_{T M}^{r}
\end{array}\right)=\Gamma\left(\begin{array}{l}
E_{T E}^{i} \\
E_{T M}^{i}
\end{array}\right) \\
& \left(\begin{array}{l}
E_{T E}^{t} \\
E_{T M}^{t}
\end{array}\right)=\mathbf{T}\left(\begin{array}{l}
E_{T E}^{i} \\
E_{T M}^{i}
\end{array}\right)
\end{aligned}
$$

Equations (14) and (15) are the ultimate solutions and are able to be put into use by a computer code to solve the layered media scattering problems.

\section{Algorithm of implementation}

The proposed formulation is algorithmic and ready to use by a computer. The algorithm requests calculations which are basic matrix algebra and are embedded as basic operations in MATLAB. The only time-consuming equation to solve by numerical solution that MATLAB employs is equation (4).
For simpler and faster solution of equation (4), we use another procedure as explained subsequently.

To solve equation (4) which is a fourth order equation, in the shape of $\left|\boldsymbol{\kappa} \boldsymbol{\mu}^{-1} \mathbf{\kappa}+k_{0}^{2} \boldsymbol{\varepsilon}\right|=\sum_{n=0}^{4} a_{n} k_{z}^{n}=0$, we need to know the polynomial coefficients $a_{n}$ that could be calculated by expanding the determinant in terms of $k_{z}$. However, these coefficients are long terms and hard to determine in closed form, in general. An alternative way is to calculate the determinant for a set of five arbitrary values of $k_{z}$ namely $s_{i}, i=0, \cdots, 4$ and putting the determinant values respectively in a series like $d_{i}$. Now, the $a_{n}$ coefficients could be easily achieved by equation:

$$
\left(\begin{array}{c}
a_{0} \\
\vdots \\
a_{4}
\end{array}\right)=\left(\begin{array}{ccc}
s_{0}^{0} & \cdots & s_{0}^{4} \\
\vdots & \ddots & \vdots \\
s_{4}^{0} & \cdots & s_{4}^{4}
\end{array}\right)^{-1}\left(\begin{array}{c}
d_{0} \\
\vdots \\
d_{4}
\end{array}\right)
$$

and the solutions of $\sum_{n=0}^{4} a_{n} k_{z}^{n}=0$ which are values of $k_{z}$ may be computed by the "roots" function in MATLAB. This process is coded in a MATLAB pseudo-code in figure 2. These calculations should be separately worked out for all values of frequency and incidence angles ( $\theta$ and $\varphi$ ) in the solution domain.

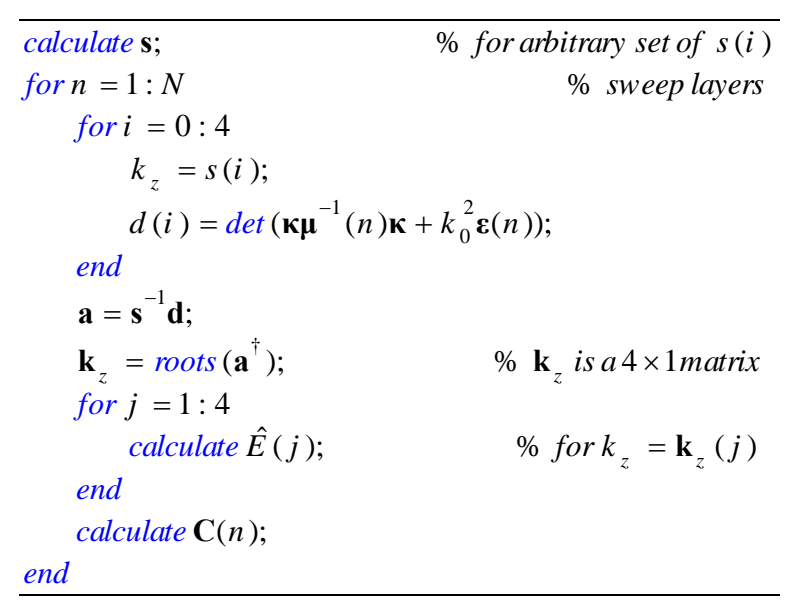

Figure 2: Pseudo-code for calculating characteristic matrices

\section{Verification of algorithm}

This section tests the validation of the proposed algorithm by different numerical examples which are chosen to reflect the importance and extension of layered media and accordingly, prove the applicability of the program. All the data applied in examples are chosen from respected papers and our results are in excellent accordance with the results in these 
references. Before presenting examples, the dispersion models which are applicable in the GUI are given.

All of media (excepting ideal vacuum), whether natural or artificial (just as metamaterials), are dispersive in the whole spectrum of electromagnetic waves because of their nonuniform spatial structure in atomic or coarser levels. Several models have been proposed to describe this electromagnetic dispersion mathematically and some of the well-known isotropic and anisotropic models for permittivity and permeability of both conventional materials and metamaterials are given in tables 1 and $2[18,19]$. To handle dispersive layers in structure, the GUI receives the parameters of these models as inputs.

Table 1: Dispersion models of permittivity

\begin{tabular}{|c|c|c|}
\hline $\begin{array}{c}\text { Model } \\
\text { dielectric }\end{array}$ & $\frac{\varepsilon_{r}}{f^{\alpha}}-j \frac{\varepsilon_{i}}{f^{\beta}}$ & $\begin{array}{c}\varepsilon_{r}, \varepsilon_{i}, \alpha, \\
\text { and } \beta\end{array}$ \\
\hline \multirow{2}{*}{$\begin{array}{c}\text { Magnetized } \\
\text { plasma }\end{array}$} & $1+\frac{f_{p}^{2}}{f_{c}^{2}-f^{2}}$ & \multirow{2}{*}{$f_{p}$ and $f_{c}$} \\
\cline { 2 - 3 } & $\pm j \frac{f_{p}^{2}\left(f_{c} / f\right)}{f_{c}^{2}-f^{2}}$ & \\
\hline $\begin{array}{c}\text { Rods } \\
\text { metamaterial }\end{array}$ & $1-\frac{f_{e p}^{2}}{f^{2}-j f \gamma_{e}}$ & \multirow{2}{*}{$f_{e p}$ and $\gamma_{e}$} \\
\hline
\end{tabular}

Table 2: Dispersion models of permeability

\begin{tabular}{|c|c|c|}
\hline \multirow{2}{*}{$\begin{array}{c}\text { Model } \\
\text { magnetic }\end{array}$} & $\frac{\mu_{r}}{f^{\alpha}}-j \frac{\mu_{i}}{f^{\beta}}$ & $\begin{array}{c}\mu_{r}, \mu_{i}, \alpha, \\
\text { and } \beta\end{array}$ \\
\hline \multirow{2}{*}{$\begin{array}{c}\text { Magnetized } \\
\text { ferrite }\end{array}$} & $1+\frac{f_{0} f_{M}}{f_{0}^{2}-f^{2}}$ & \multirow{2}{*}{$f_{0}$ and $f_{M}$} \\
\cline { 2 - 3 } & $\pm j \frac{f_{M} f}{f_{0}^{2}-f^{2}}$ & \\
\hline $\begin{array}{c}\text { Rings } \\
\text { metamaterial }\end{array}$ & $1-\frac{f_{m p}^{2}-f_{m o}^{2}}{f^{2}-f_{m o}^{2}-j f \gamma_{m}}$ & $\begin{array}{c}f_{m o}, f_{m p}, \\
\text { and } \gamma_{m}\end{array}$ \\
\hline
\end{tabular}

\subsection{PEC with magnetoplasma coating}

The first example is taken from [15] in which the transfer matrix method is utilized to calculate the reflection coefficient of a conductor with a magnetized plasma layer that is biased with a z-direction magnetic field. Magnetized plasmas and magnetized ferrites are used in microwave engineering to fabricate non-reciprocal components which need anisotropic media [19]. Elements of relative permittivity are assumed to be $\varepsilon_{x x}=\varepsilon_{y y}=40, \varepsilon_{x y}=-\varepsilon_{y x}=80 j$, $\varepsilon_{z z}=1$, and zero for others. The GUI layout and the problem's solution for $4<k_{0} d<6$ is plotted in Figure 3 .
The solution is in agreement with original article. However, the transfer matrix method fails for large values of $k_{0} d$ due to round-off errors as enormous exponential terms arise. For these values, other methods like recursive methods should be used.

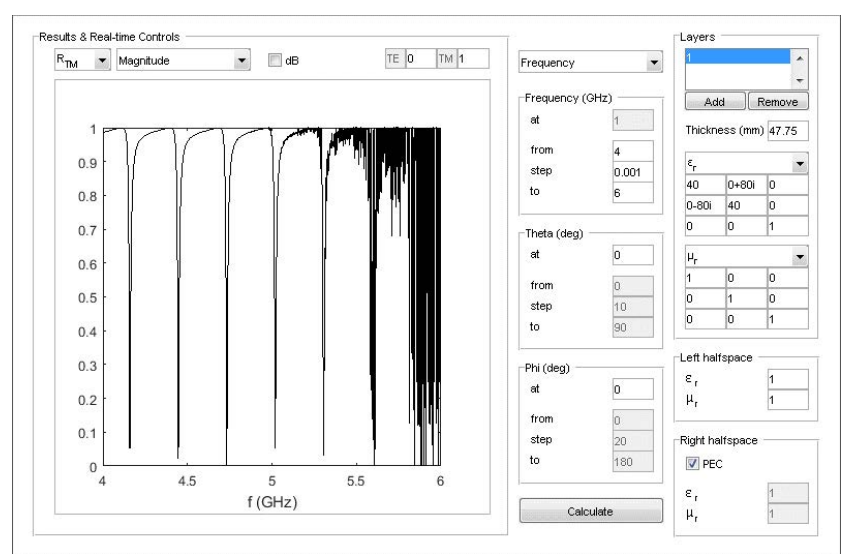

Figure 3: The GUI layout and its response for the first example. This example demonstrates the accuracy of the proposed algorithm for smaller $k_{0} d$ values and its unstable response for higher frequencies.

\subsection{Dispersive DPS-DNG bilayer structure}

One great advantage of our algorithm is that its solution is valid for any sign of permittivity and permeability elements without extra considerations. In fact, the solutions of (4) satisfy the criteria of choosing correct sign [4] when the medium has negative values in its $\boldsymbol{\varepsilon}$ or $\boldsymbol{\mu}$. Therefore, we are able to analyze any class of DPS (double positive), DNG (double negative), ENG (epsilon negative), and MNG (mu negative) materials with the same equations. Combining these media in one structure, yields several interesting cases like absolute transparency.

Figure 4 shows the reflectance (reflected wave power normalized to incident power) against frequency from a pair of DPS and DNG isotropic layers with thicknesses 1 and 2 millimeters that acts as a backward notch filter. The first layer is assumed to be a non-magnetic material with relative permittivity of 2.2 and the second layer is a lossless metamaterial with dispersion relations of table 1 . Dispersion parameters are given in the figure legend. Agreement with [5] proves the potential of the algorithm to analyze highly dispersive metamaterial structures.

\subsection{Perfectly matched layer}

Perfectly matched layer (PML) is an extremely lossy medium that is used to absorb radiation in boundaries of numerical electromagnetic problems (mostly when finite difference time domain is adopted) to make the simulation space finite. The most common type of these media is Gedney's PML [20] which is defined as a uniaxial anisotropic material with permittivity elements of $\varepsilon_{x x}=\varepsilon_{y y}=1 / \varepsilon_{z z}=1+\sigma / j \omega \varepsilon_{0}$ 
and zero elsewhere and the same values for permeability. For $\sigma / \omega \varepsilon_{0} \gg 1$ (which is always true in PMLs), we have $1+\sigma / j \omega \varepsilon_{0} \cong \sigma / j \omega \varepsilon_{0}$ and the permittivity and permeability become entirely imaginary. Therefore, we may use lossy electric and lossy magnetic models of tables 1 and 2 with $\varepsilon_{i}=\mu_{i}=\sigma / 2 \pi \varepsilon_{0}$ and $\beta=1$ for $\mathrm{xx}$ and $\mathrm{yy}$ elements and $\varepsilon_{i}=\mu_{i}=-2 \pi \varepsilon_{0} / \sigma$ and $\beta=-1$ for $\mathrm{zz}$ elements to model the Gedney's PML. Gedney's PML is matched to the air for any incidence angle, so is able to make better absorption relative to ordinary isotropic absorber with $\varepsilon=\mu=1+\sigma / j \omega \varepsilon_{0}$ parameters-which is perfectly matched only for normal incidence-when the incidence angle is not zero. However, for the normal incidence, the two cases are identical for equal conductivities. Figure 5 compares the isotropic and anisotropic (Gedney’s) absorbers' power for killing TE-polarized electromagnetic wave with frequencies between 0-10 GHz and at angles of 0-90 degrees when they are backed by PEC. The layer's thickness and conductivity are assumed to be $1(\mathrm{~cm})$ and $1(\mathrm{~S} / \mathrm{m})$. While the two act excellent for normal incidence, the anisotropic case is more efficient for oblique waves (as it can be seen from its concavity).

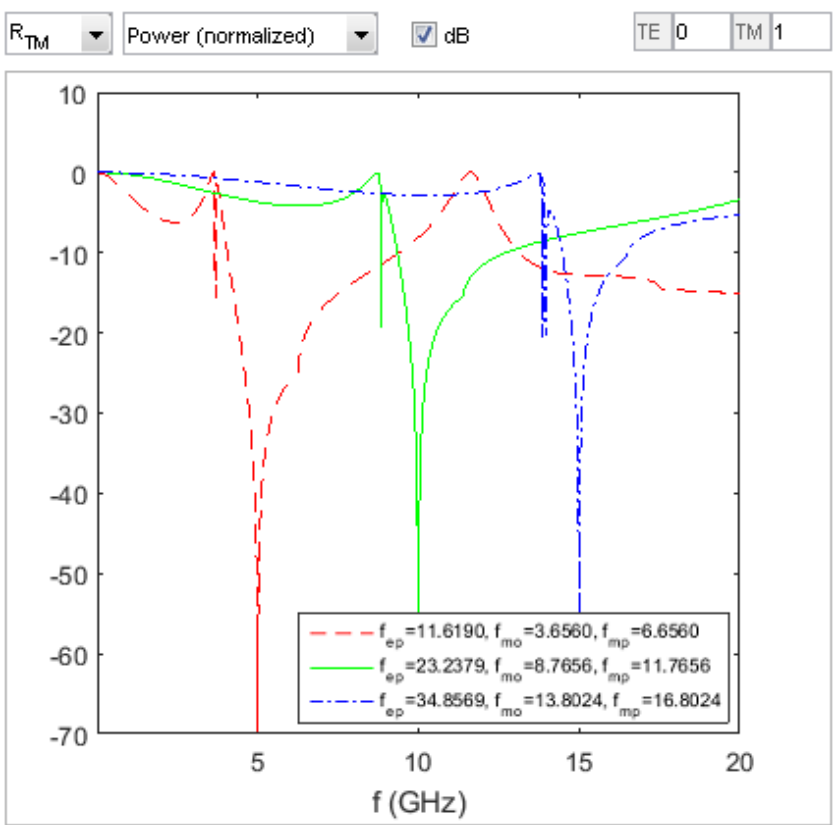

Figure 4: Reflectance of wave with TM polarization and angle $45^{\circ}$ from a dispersive DPS-DNG bilayer structure with different dispersion parameters given in the legend (in $\mathrm{GHz}$ ) in terms of $\mathrm{dB}$
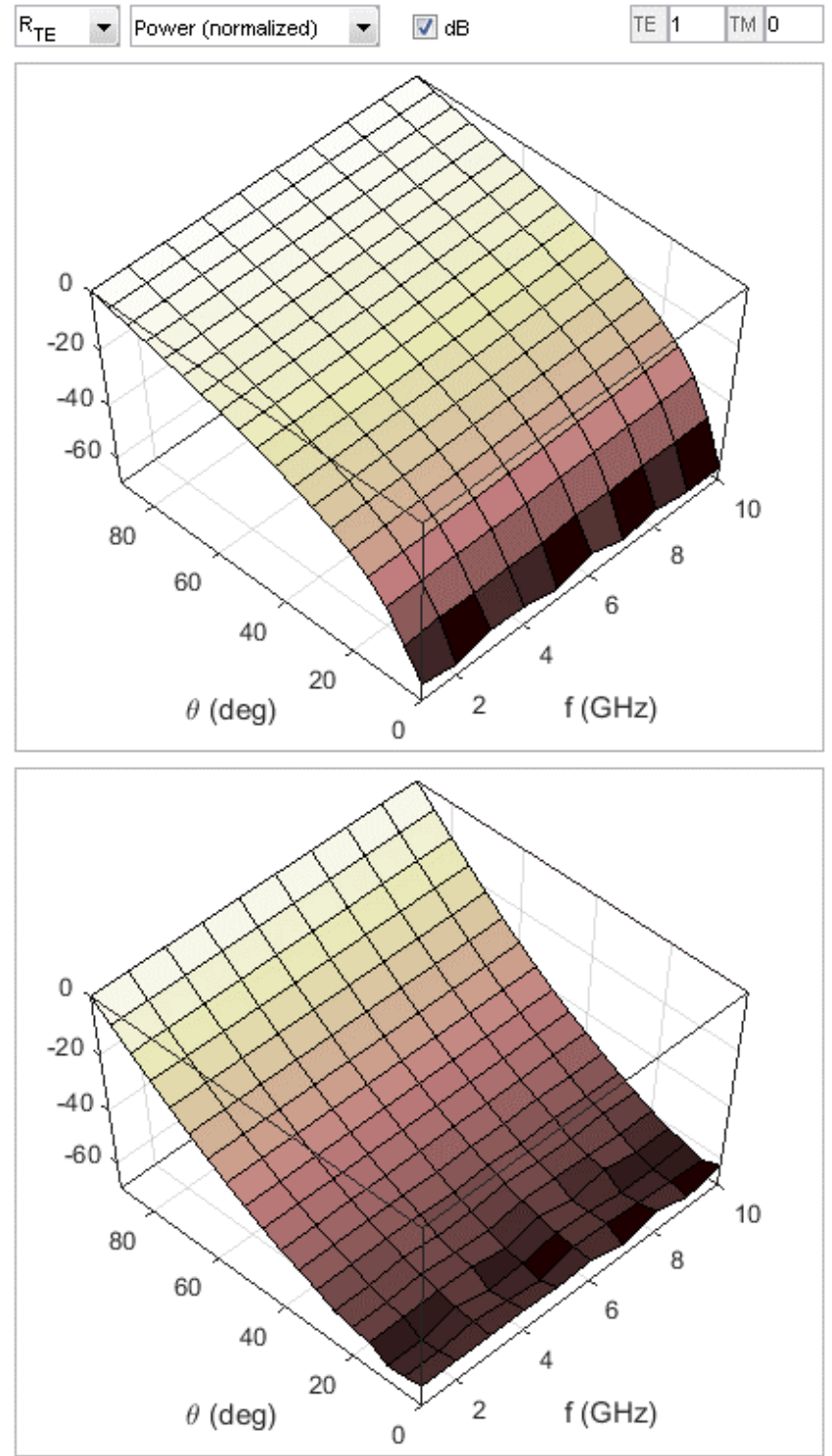

Figure 5: Reflectance of TE-polarized wave from perfect conductor covered with isotropic (top) and anisotropic (bottom) perfectly matched layers against frequency and incidence angle

\section{Conclusions}

First, mathematical formulation based on the transfer matrix method for planar electromagnetic wave scattering analysis of planar layered structures consisting homogeneous materials that can be lossy, anisotropic, dispersive, and metamaterial was presented. The mathematical formulation was optimized for being coded by MATLAB scripting language and for easy and standalone use of the code, it was packaged as a graphical user interface. After considering the algorithm, the program was validated by three examples, and the good agreement with reports in resources, proved the integrity of the program. 


\section{References}

[1] R. Levy, R.V. Snyder, G. Matthaei, Design of microwave filters, IEEE Trans. Microwave Theory Tech. 50(3): 783793, 2002.

[2] I. Moreno, J.J. Araiza, M. Avendano-Alejo, Thin-film spatial filters, Opt. Lett. 30(8): 914-916, 2005.

[3] Y. Huang, Y. Feng, T. Jiang, Electromagnetic cloaking by layered structure of homogeneous isotropic materials, Opt. Exp. 15(18): 11133-11141, 2007.

[4] H. Oraizi, A. Abdolali, Design and optimization of planar multilayer antireflection metamaterial coatings at $\mathrm{Ku}$ band under circularly polarized oblique plane wave incidence, Prog. Electromagn. Res. C 3: 1-18, 2008.

[5] H. Oraizi, A. Abdolali, Mathematical formulation for zero reflection from multilayered metamaterial structures, IET Microw. Antennas Propag. 3(6): 987996, 2009.

[6] A. Mirala, A. Abdolali, Zero reflection from layered anisotropic metamaterial structures, Waves Random Complex Media 23(2): 152-168, 2013.

[7] M. Al Shakhs, L. Augusto, L. Markley, K.J. Chau, Boosting the Transparency of Thin Layers by Coatings of Opposing Susceptibility: How Metals Help See Through Dielectrics, Scientific Reports 6: 20659, 2016.

[8] C. Sabah, S. Uckun, High reflection coating with negative and positive refractive indices, Piers Online 5(7): 601-604, 2009.

[9] H. Maftooli, S.H.H. Sadeghi, R. Moini, H. Karami, Time-domain electromagnetic analysis of multilayer structures using the surface equivalent principle and mixed potential integral equations, IEEE Trans. Microw. Theory Techn. 63(1): 99-106, 2015.

[10] P. Yeh, Optics of anisotropic layered media: A new $4 \times 4$ matrix algebra, Surface Science 96: 41-53, 1980.

[11] J. Hao, L. Zhou, Electromagnetic wave scatterings by anisotropic metamaterials: Generalized $4 \times 4$ transfermatrix method, Phys. Rev. B 77: 094201, 2008.

[12] Y. Fang, L. Wu, J. Zhang, Excitation of plane waves for FDTD analysis of anisotropic layered media, IEEE Antennas Wireless Propag. Lett. 8: 414-417, 2009.

[13] K.A. Michalski, J.R. Mosig, Multilayered media Green's functions in integral equation formulations, IEEE Trans. Antennas Propag. 45(3): 508-519, 1997.

[14]H. Oraizi, M. Afsahi, Analysis of planar dielectric multilayers as FSS by transmission line transfer matrix method (TLTMM), Prog. Electromagn. Res. 74: 217240, 2007.

[15] H.D. Yang, A spectral recursive transformation method for electromagnetic waves in generalized anisotropic media, IEEE Trans. Antennas Propag. 45(3): 520-526, 1997.

[16] M. Heidary, A. Abdolali, M.M. Salary, An exact formulation for the reflection coefficient from anisotropic multilayer structures with arbitrary backing, Progress In Electromagnetics Research M 30: 79-93, 2013.

[17]A. Eroglu, Wave Propagation and Radiation in Gyrotropic and Anisotropic Media, lst ed., Springer, New York, 2010.
[18]H. Oraizi, A. Abdolali, Several theorems for reflection and transmission coefficients of plane wave incidence on planar multilayer metamaterial structures, IET Microw. Antennas Propag. 4(11): 1870-1879, 2010.

[19] K. Zhang, D. Li, Electromagnetic theory for microwaves and optoelectronics, 2nd ed., Springer, Berlin, 2007.

[20] S. Gedney, An anisotropic perfectly matched layerabsorbing medium for the truncation of FDTD-lattices, IEEE Trans. Antennas Propagat. 44(12): 1630-1639, 1996. 